\title{
Novel homozygous ALS2 nonsense mutation (p.GIn715X) in sibs with infantile-onset ascending spastic paralysis: the first cases from northwestern Europe
}

\author{
Corien C Verschuuren-Bemelmans ${ }^{1}$, Pia Winter ${ }^{2}$, Deborah A Sival ${ }^{3}$, Jan-Willem Elting ${ }^{4}$, \\ Oebele F Brouwer ${ }^{4}$ and Ulrich Müller*,2 \\ ${ }^{1}$ Department of Genetics, University Medical Center Groningen, University of Groningen, Groningen, The Netherlands;
${ }^{2}$ Institute of Human Genetics, Justus-Liebig-University, Giessen, Germany; ${ }^{3}$ Department of Pediatrics, University
Medical Center Groningen, University of Groningen, Groningen, The Netherlands; ${ }^{4}$ Department of Neurology,
University Medical Center Groningen, University of Groningen, Groningen, The Netherlands
}

We describe a previously not recognized nonsense mutation in exon 10 of the ALS2 gene in two sibs with infantile-onset ascending spastic paralysis. The mutation predicts chain termination at amino-acid position 715 of the gene product ALSIN (p.GIn715X). The sibs' parents are descendants of a common ancestor who lived in the northern Netherlands during the eighteenth century. This is the first ALS2 mutation detected in northwestern Europeans. The findings emphasize that mutations in ALS2 also need to be considered in patients from northwestern Europe with early-onset spastic paralysis and amyotrophic or primary lateral sclerosis.

European Journal of Human Genetics (2008) 16, 1407-1411; doi:10.1038/ejhg.2008.108; published online 4 June 2008

Keywords: IAHSP; JPLS; ALS2; ALSIN; homozygous nonsense mutation

\section{Introduction}

Homozygous mutations in the ALS2 gene can result in one of three clinically distinct motor neuron diseases: (1) autosomal recessive juvenile amyotrophic lateral sclerosis (ALS2, OMIM no. 205100), (2) juvenile primary lateral sclerosis (JPLS, OMIM no. 606353), or (3) infantile-onset ascending spastic paralysis (IAHSP, OMIM no. 607225). ${ }^{1-4}$ In ALS2 both upper and lower motor neurons are affected, whereas neurodegeneration only involves upper motor neurons in JPLS and IAHSP. ${ }^{5,6}$ Despite this difference in neuropathology, there is considerable clinical overlap of the major signs and symptoms in the three conditions,

*Correspondence: Professor U Müller, Institut für Humangenetik, JustusLiebig-Universität, Schlangenzahl 14, Giessen 35392, Germany.

Tel: + 49641994 1600; Fax: + 49641994 1609;

E-mail: ulrich.mueller@humangenetik.med.uni-giessen.de

Received 20 February 2008; revised 29 April 2008; accepted 6 May 2008; published online 4 June 2008 including spasticity of the limbs and dysarthria. The age of onset is during early childhood (first or second year), in the three disorders but appears to be somewhat later in ALS2 than in JPLS and IAHSP.

The ALS2 gene is located on the long arm of chromosome 2 (2q33) and is composed of 34 exons. ${ }^{1,2}$ It can be transcribed into two alternative transcripts of 6.5 and $2.6 \mathrm{~kb}$. The large transcript encodes ALSIN, a protein of $184 \mathrm{kD}$. ALSIN contains three putative guanine nucleotide exchange factor (GEF) domains, the N-terminal regulator of chromatin condensation (RCC1) domain, the central Db1 and pleckstrin homology (DH/PH) domains, and the C-terminal vacuolar protein sorting 9 (VPS9) domain. The RCC1 domain is characteristic of a GEF for GTPase Ran, ${ }^{7}$ the $\mathrm{DH} / \mathrm{PH}$ domains act as Rho GEFs, ${ }^{8}$ and the VPS9 domain interacts with endosome-associated small G protein Rab5a. ${ }^{9}$ This observation and experimental evidence points to a function of ALSIN as a GEF protein. 
It is not known whether the short transcript is physiologically translated into a functional polypeptide as well.

To date, a total of 12 different mutations have been described in the ALS2 gene. All had occurred in people from Mediterranean countries and from Asia. Here we describe the first mutation in ALS2 in sibs from northwestern Europe. They are the children of consanguineous parents, who are descendants of a common ancestor who lived in the eighteenth century (around 1750) in the province of Friesland, in the northern part of the Netherlands. The findings are discussed in the context of published ALS2 mutations.

\section{Materials and methods}

DNA extraction and sequencing of the 34 exons of ALS2 were performed according to standard procedures.

\section{Case reports}

Patient 1, the proband, is the first child of healthy parents of Dutch descent. She was born at term after an uncomplicated pregnancy and delivery. At the age of 8 months she developed distal spasticity in her legs and axial hypotonia. She was never able to sit or stand without support. During the following 3 years, spastic diplegia slowly progressed to the upper extremities. Starting at age 5 , she developed tetraplegia; with the lower extremities being more severely affected than the upper extremities, soft pseudobulbar speech, and dysphagia. She has never achieved bowel or bladder control. Now aged 13 years, she suffers from drooling, receives gastroenteric feeding by Mickey button and speech has become anarthric. She is fully wheelchair dependent and voluntary movements of the hands are hampered by sudden hyperextension. Cognition, behavior, vision, and hearing have remained within the normal range.

Patient 2, the brother of the proband, is the third child born to the same parents. He was born at term, after an uncomplicated pregnancy and delivery. He developed normal gross and fine motor function during his first 18 months. He was able to grasp, sit, stand (without support), and walk (with support). However, from then onward, he progressively developed spastic diplegia with scissoring at the hips and his speech became nasal. From the age of 4 years, the volume of his voice decreased and swallowing and coughing deteriorated. His fine motor skills were assessed as being below average. He has never gained voluntary control over bladder and bowel function. Now aged 8 years, he is still able to walk short distances (walkerassisted) and he is still capable of deliberate hand movements. Like his elder sister, his cognition, behavior, vision, and hearing remain within the normal range.

In both children, neurological examination showed undisturbed extrapyramidal, lower motor neuron, and sensory function. Additional studies showed that motor and sensory conduction of peripheral nerves were normal, and needle electromyography did not reveal myopathic or neuropathic abnormalities. However, motor-evoked potentials were completely unobtainable after cortical stimulation, in either arm or leg muscles. These findings confirmed the presence of severe upper motor neuron dysfunction and absence of lower motor neuron involvement. Cerebral MRIs and blood tests (including lactate and lysosomal enzymes) were normal. On physical examination the parents showed no signs of motor neuron dysfunction, and their second child is healthy.

\begin{abstract}
Molecular findings
We first sequenced all 34 exons and adjacent intronic portions of $A L S 2$ in one of the two index cases. We found ten homozygous sequence variants, four of which were in exons and six in introns (Table 1). Of these sequence changes, nine have been described before and are considered neutral single nucleotide polymorphisms (SNPs). One sequence change (c.2143C $>\mathrm{T}$ ) in exon 10, however, was predicted to result in the generation of a stop codon at amino-acid position 715 (p.Gln715X) (Figure 1). We then sequenced exon 10 and those exons and introns that
\end{abstract}

Table 1 Sequence changes observed in ALS2 in the patients and their parents

\begin{tabular}{|c|c|c|c|c|c|c|c|}
\hline Exon/Intron & SNP ID & Ref SNP(rs no.) & Effect & Genotype father & Genotype mother & Genotype sib 1 & Genotype sib 2 \\
\hline Exon 4 & c. $1102 \mathrm{G}>\mathrm{A}$ & rs3219156 & Val368Met & Homozygous & Homozygous & Homozygous & Homozygous \\
\hline Intron 10 & IVS10-62C >T & rs3731703 & & Heterozygous & Homozygous & Homozygous & Homozygous \\
\hline Exon 10 & c. $2143 C>T$ & & Gln 715 stop & Heterozygous & Heterozygous & Homozygous & Homozygous \\
\hline Exon 13 & c. $2466 \mathrm{G}>\mathrm{A}$ & rs2276615 & Val822 Val & Heterozygous & Homozygous & Homozygous & Homozygous \\
\hline Intron 25 & IVS $25+25 C>T$ & rs3219167 & & Heterozygous & Homozygous & Homozygous & Homozygous \\
\hline Intron 25 & IVS25-76T $>C$ & rs 2882231 & & Heterozygous & Homozygous & Homozygous & Homozygous \\
\hline Exon 26 & c. $4015 \mathrm{C}>\mathrm{T}$ & rs3219168 & Leu1339Leu & Heterozygous & Homozygous & Homozygous & Homozygous \\
\hline Intron 26 & IVS26-64G >A & rs1210940 & & Homozygous & Homozygous & Homozygous & Homozygous \\
\hline Intron 29 & IVS29-48T >C & rs3219170 & & Heterozygous & Homozygous & Homozygous & Homozygous \\
\hline Intron 30 & IVS30-69T >A & rs3219171 & & Heterozygous & Homozygous & Homozygous & Homozygous \\
\hline
\end{tabular}

Abbreviation: SNPs, single nucleotide polymorphisms.

The nonsense mutation in exon 10 is highlighted. Bold type indicates SNPs resulting in amino-acid changes. 


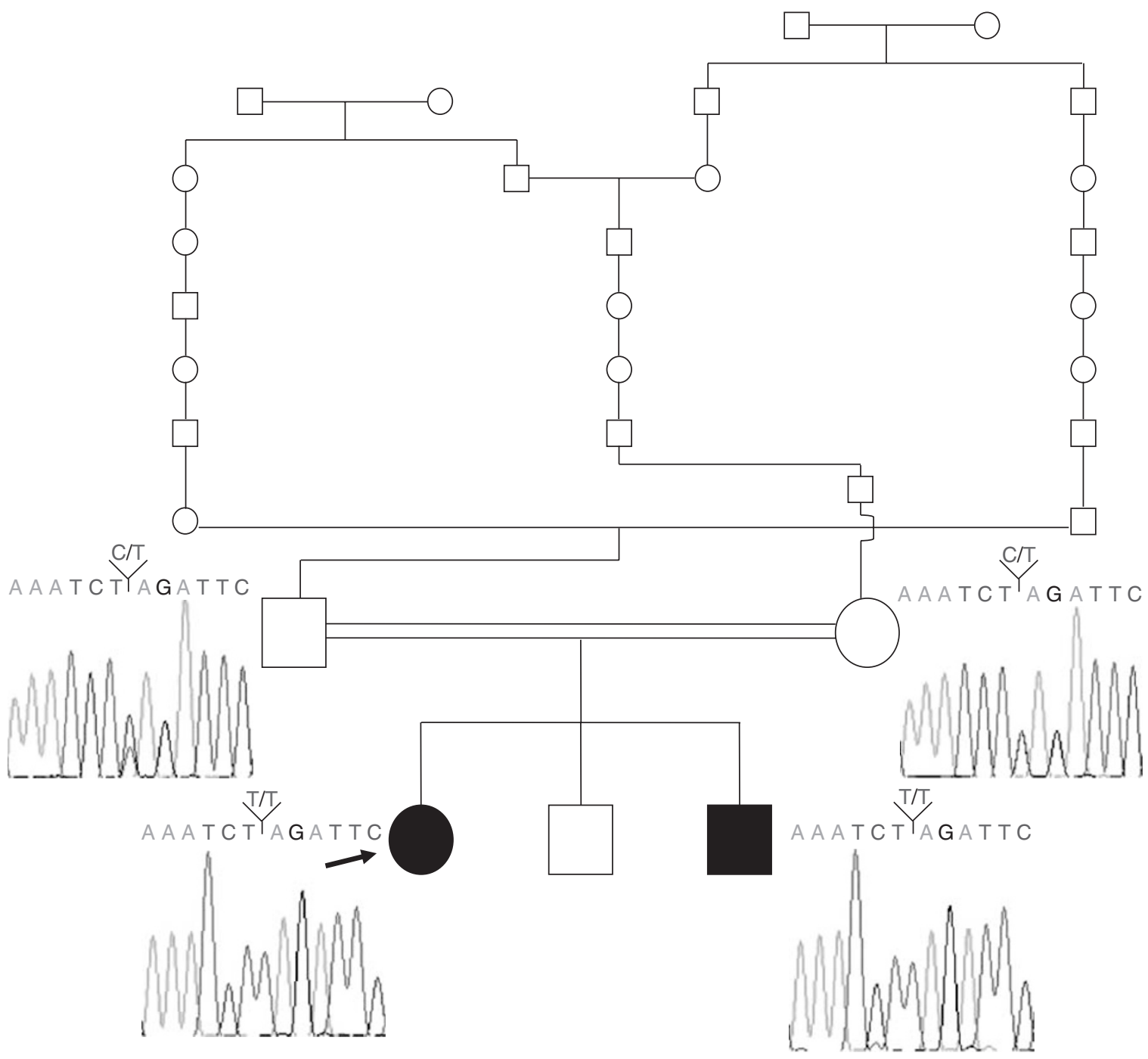

Figure 1 Pedigree of the family. The index case is marked by arrow. Sequence chromatographs are shown of both patients and their parents (for details see text).

contain SNPs in the affected sib and the patients' parents. Although the causative mutation (c.2143C $>$ T) and the SNPs are homozygous in both sibs, the parents were heterozygous for the mutation and the father was heterozygous for most of the SNPs (Figure 1; Table 1). The mother was homozygous for all SNPs. The mutation only affects the long transcript of ALS2. It predicts truncation of ALSIN and the absence of most of the $\mathrm{DH} / \mathrm{PH}$ domains, the eight-membrane occupation and recognition nexus motifs (MORN), and the VPS9 domain.

\section{Discussion}

We report on two sibs with clinical features of infantileonset ascending hereditary spastic paralysis (IAHSP). The ALS2 mutation found in these children is the first one discovered in northwestern Europeans. All previously detected ALS2 mutations were found in people from countries surrounding the Mediterranean and from Asia (Table 2). The mutation, a previously not recognized nonsense mutation, most likely originates from one of the common ancestors of the patients' parents at least 250 years ago (Figure 1). It is the second nonsense mutation found in the ALS2 gene. The nonsense mutation previously reported was in exon 18 (p.Arg998X) in two sisters with IAHSP from a Buchari Jewish family. ${ }^{13}$ Altogether there are now 13 ALS2 mutations known (Table 2).

As is evident from Table 2, there is no clear-cut genotype-phenotype correlation. However, ALS2 has not been found in persons with truncated ALSIN consisting of more than the amino acids encoded by the first four/five exons. The significance of this observation is not clear. When the first mutations were discovered in ALS2, it was 
Table 2 Mutations in ALS2, IAHSP, and JPLS

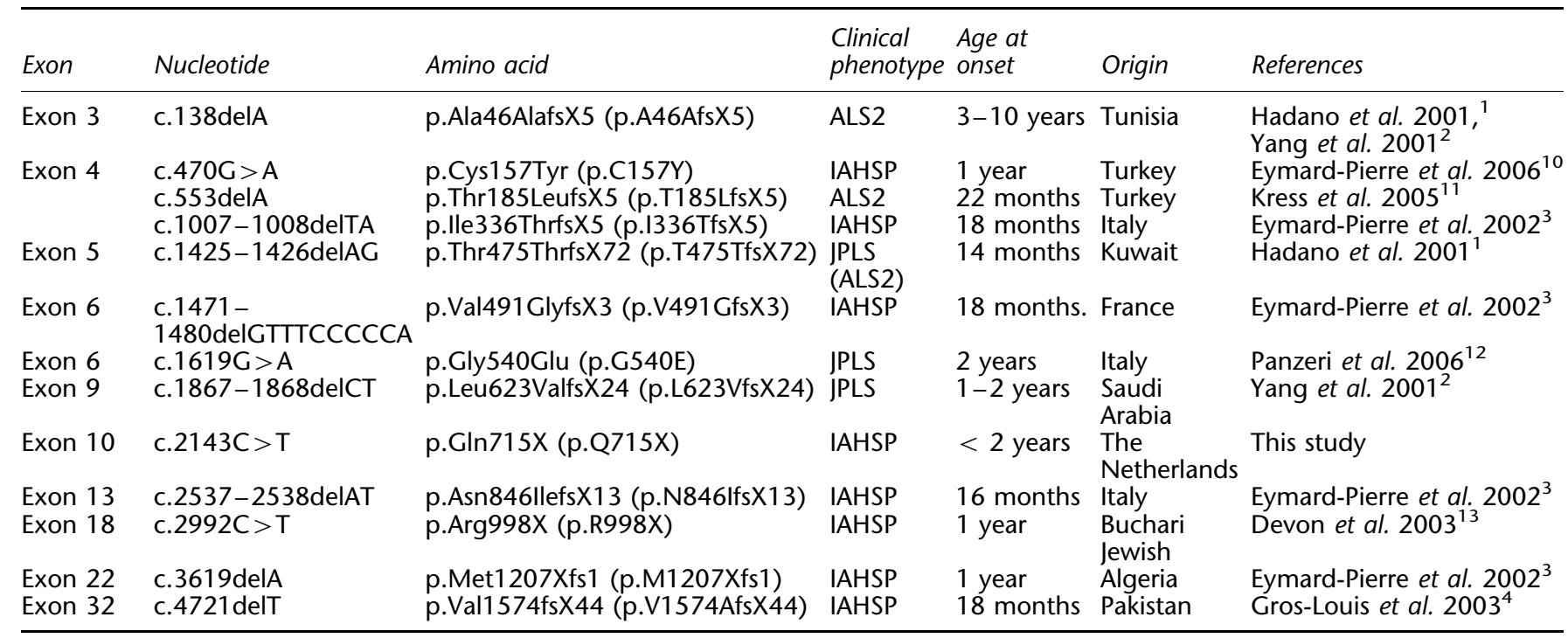

Abbreviations: ALS, amyotrophic lateral sclerosis; IAHSP, infantile-onset ascending spastic paralysis; JPLS, juvenile primary lateral sclerosis.

Nomenclature for nucleotide position according to Antonarakis (1998) ${ }^{17}$ with A of start codon ATG within exon 2 being the first nucleotide. Note that some investigators calculate mutations from the A of an ATG within the untranslated exon 1 of ALS2. The start codon in exon 2 and the ATG within exon 1 are separated by 123 bp of exonic DNA.

thought that lower motor neurons were protected by the small transcript of ALS2. ${ }^{1}$ Accordingly, ALS2 with both upper and lower motor neurons affected should always occur when exons encoding this transcript (exons 1-4) are affected. This is not the case, as IAHSP was found in a 24-year-old man with a homozygous $2 \mathrm{bp}$ deletion in exon 4 (see table; reference ${ }^{3}$ ) interfering with both the long and the small transcripts. It is not yet understood why mutations affecting similar parts of ALSIN can result in either ALS2, JPLS, or IAHSP. It is also unclear why the same phenotype can occur in patients with different parts of ALSIN affected. It is likely that additional genes (modifier genes), and possibly environmental influences, shape the phenotype.

The mutation spectrum observed in ALS2 shows that the VPS9 domain is important in normal ALSIN function. This domain is affected in all cases that result in truncation of ALSIN. Its loss alone can give rise to either JPLS or IAHSP. By binding to GTPase RAB5, VPS9 functions as a GEF for this GTPase. Furthermore, the VPS9 domain mediates binding of ALSIN to endosomes. ${ }^{9}$ It is important in endosome fusion and trafficking. ${ }^{14}$ Therefore, disturbance of normal endosome function, in addition to loss of GEF activity for RAB5, might be the underlying cause of motor neuron degeneration in patients with ALS2 mutations. Missense mutations involving other domains can also cause motor neuron degeneration (Table 2). However, both the missense mutations found so far disable ALSIN function, including its VPS9 domain. Mutation of p.Gly540Glu lies within the RCC1 domain, results in delocalization of ALSIN within the cell, and appears to be neurotoxic. ${ }^{12}$ The p.Cys157Tyr mutation is also located within the RCC1 domain and causes instability of the mutant ALSIN. ${ }^{10}$ This observation supports the idea of Topp et $a l^{15}$ who assumed the RCC1 domain played a structural rather than an enzymatic role in ALSIN. Furthermore, the relatively poor evolutionary conservation of the N-terminal region of ALSIN is also consistent with a structural rather than an important metabolic function of RCC1. ${ }^{16}$

In conclusion, we have described a nonsense mutation in exon 10 of the ALS2 gene in two sibs of northwestern European origin. It is very likely that the mutation can be traced back to a common ancestor of the patients' parents who lived in the northern part of the Netherlands around 1750. Thus, mutations in the ALS2 gene must also be considered in children with juvenile ALS, JPLS, and IAHSP from northwestern Europe, even when there is no immediate suspicion of parental consanguinity.

\section{Acknowledgements}

We thank Jackie Senior and Mary Kay Steen-Müller for editing the text and Jan J Hoetjes for his extensive genealogy work.

\section{References}

1 Hadano S, Hand CK, Osuga $\mathrm{H}$ et al: A gene encoding a putative GTPase regulator is mutated in familial amyotrophic lateral sclerosis 2. Nat Genet 2001; 29: 166-173.

2 Yang Y, Hentati A, Deng HX et al: The gene encoding alsin, a protein with three guanine-nucleotide exchange factor domains, is mutated in a form of recessive amyotrophic lateral sclerosis. Nat Genet 2001; 29: 160-165. 
3 Eymard-Pierre E, Lesca G, Dollet S et al: Infantile-onset ascending hereditary spastic paralysis is associated with mutations in the alsin gene. Am J Hum Genet 2002; 71: 518-527.

4 Gros-Louis F, Meijer IA, Hand CK et al: An ALS2 gene mutation causes hereditary spastic paraplegia in a Pakistani kindred. Ann Neurol 2003; 53: 144-145.

5 Pringle CE, Hudson AJ, Munoz DG et al: Primary lateral sclerosis, clinical features, neuropathology and diagnostic criteria. Brain 1992; 115: 495-520.

6 Lesca G, Eymard-Pierre E, Santorelli FM et al: Infantile ascendine hereditary spastic paralysis (IAHSP): clinical features in 11 families. Neurology 2003; 60: 674-682.

7 Ohtsubo M, Kai R, Furuno $\mathrm{N}$ et al: Isolation and characterization of the active cDNA of the human cell cycle gene (RCC1) involved in the regulation of onset of chromosome condensation. Genes Dev 1987; 1: 585-593.

8 Schmidt A, Hall A: Guanine nucleotide exchange factors for Rho GTPases: turning on the switch. Genes Dev 2002; 16: 1587-1609.

9 Otomo A, Hadano S, Okada T et al: ALS2, a novel guanine nucleotide exchange factor for the small GTPase Rab5, is implicated in endosomal dynamics. Hum Mol Genet 2003; 12: 1671-1687.

10 Eymard-Pierre E, Yamanaka K, Haeussler M et al: Novel missense mutation in ALS2 gene results in infantile ascending hereditary spastic paralysis. Ann Neurol 2006; 59: 976-980.
11 Kress JA, Kühnlein P, Winter P et al: Novel mutation in the ALS2 gene in juvenile amyotrophic lateral sclerosis. Ann Neurol 2005; 58: $800-803$.

12 Panzeri C, De Palma C, Martinuzzi A et al: The first ALS2 missense mutation associated with JPLS reveals new aspects of alsin biological function. Brain 2006; 129: 1710-1719.

13 Devon RS, Helm JR, Rouleau GA et al: The first nonsense mutation in alsin results in a homogeneous phenotype of infantile-onset ascending spastic paralysis with bulbar involvement in two siblings. Clin Genet 2003; 64: 210-215.

14 Hadano S, Kunita R, Otomo A et al: Molecular and cellular function of ALS2/alsin: Implication of membrane dynamics in neuronal development and degeneration. Neurochem Int 2007; 51: $74-84$.

15 Topp J, Gray NW, Gerard RD et al: Alsin is a Rab5 and Rac1 guanine nucleotide exchange factor. J Biol Chem 2004; 23 : $24612-24623$

16 Devon RS, Schwab C, Topp JD et al: Cross-species characterization of the ALS2 gene and analysis of its pattern of expression in development and adulthood. Neurobiol Dis 2005; 18: $243-257$.

17 Antonarakis SE, The Nomenclature Working Group: Recommendations for a nomenclature system for human gene mutations. Hum Mut 1998; 11: 1-3. 